\title{
Sistemas de preparo do solo, plantas de cobertura e produtividade da cultura da mandioca
}

\author{
Auro Akio Otsubo ${ }^{(1)}$, Fábio Martins Mercante ${ }^{(1)}$, Rogério Ferreira da Silva ${ }^{(2)}$ e Clovis Daniel Borges ${ }^{(2)}$
}

\begin{abstract}
(1)Embrapa Agropecuária Oeste, Caixa Postal 661, CEP 79804-970 Dourados, MS. E-mail: auro@cpao.embrapa.br, mercante@cpao.embrapa.br (2) Centro Universitário da Grande Dourados, Rua Balbina de Matos, № 2121, CEP 79824-900 Dourados, MS. E-mail: rogerio@uems.br, clovisdb@yahoo.com.br
\end{abstract}

Resumo - O objetivo deste trabalho foi avaliar os efeitos do uso de plantas de cobertura e de sistemas de preparo do solo, no desenvolvimento e na produtividade da cultura da mandioca (Manihot esculenta Crantz). $\mathrm{O}$ trabalho foi conduzido em Argissolo Vermelho, sob sistema convencional de preparo do solo, e em cultivo mínimo sobre palhada de mucuna-cinza (Stizolobium cinereum Piper \& Tracy), sorgo granífero [Sorghum bicolor (L.) Moench] e milheto [Pennisetum americanum (L.) K. Schum.]. Aos dezoito meses após o plantio da mandioca, foram avaliados: altura de plantas, produção de massa de matéria seca da parte aérea, número de raízes tuberosas, produtividade, percentagem de matéria seca e de amido nas raízes tuberosas e índice de colheita. Observou-se que o sistema convencional de preparo do solo pode ser substituído, na cultura da mandioca, pela prática do cultivo mínimo, associada ao uso de coberturas vegetais, por promover incrementos significativos na produtividade da cultura, especialmente, quando se utiliza o milheto como planta de cobertura. O uso de plantas de cobertura no pré-cultivo de mandioca, em sistema de preparo mínimo do solo, representa uma alternativa eficiente para um melhor manejo dessa cultura.

Termos para indexação: Manihot esculenta, Pennisetum americanum, Sorghum bicolor, Stizolobium cinereum, cultivo mínimo, manejo do solo.

\section{Soil tillage systems, cover crops and productivity in cassava}

\begin{abstract}
The objective of this work was to evaluate the effects of cover crops and soil tillage systems in the development and yield of cassava (Manihot esculenta Crantz). The experiment was carried out in an Arenic Hapludult under conventional tillage, and in a minimum tillage system over mucuna (Stizolobium cinereum Piper \& Tracy), sorghum [Sorghum bicolor (L.) Moench] and millet straw [Pennisetum americanum (L.) K. Schum.]. Eighteen months after cassava planting, the following variables were evaluated: plant height, shoot dry matter production, number of roots, yield, dry matter and starch content on storage roots, and harvest index. It was observed that conventional tillage could be replaced by minimum tillage in cassava crop, when associated to cover crops use, since it leads to a significant increase in cassava yield, particularly when using millet as cover crop. The use of cover crops before cassava cultivation, in a minimum tillage system, is an efficient alternative for a better crop management.

Index terms: Manihot esculenta, Pennisetum americanum, Sorghum bicolor, Stizolobium cinereum, minimum tillage, soil management.
\end{abstract}

\section{Introdução}

A mandioca (Manihot esculenta Crantz) é uma cultura de grande importância socioeconômica, cultivada em todas as regiões brasileiras nas mais diversas condições edafo-climáticas. É uma das principais fontes de calorias para populações de países tropicais, importante matéria-prima para agroindústrias e geradora de emprego e renda, principalmente para pequenos produtores (Cardoso, 2003).
Nessa cultura, o preparo do solo é tradicionalmente realizado com uma aração e duas gradagens, o que pode trazer problemas de erosão, compactação e empobrecimento progressivo do solo, com conseqüente diminuição da produtividade dos cultivos (Gabriel Filho et al., 2000). O crescimento inicial lento, o grande espaçamento entre plantas, a necessidade de capinas durante os primeiros estádios da cultura, e a 
movimentação do solo por duas vezes a cada ciclo (no plantio e na colheita) são características agronômicas dessa cultura que podem deixar o solo descoberto e desprotegido de dois a três meses após o plantio e, conseqüentemente, provocar grandes perdas de solo e água por erosão (Souza et al., 2006). O plantio direto ou o cultivo mínimo associado ao uso de plantas de cobertura do solo podem aumentar a sustentabilidade dos sistemas de produção de mandioca, além de oferecer condições mais favoráveis ao crescimento e desenvolvimento das plantas.

A utilização de práticas conservacionistas de manejo do solo tem recebido grande ênfase da pesquisa atualmente, basicamente no que se refere à manutenção e à melhoria das propriedades físicas, químicas e biológicas dos solos cultivados e às suas implicações no rendimento das culturas. Essas técnicas propiciam condições mais favoráveis para a manutenção e recuperação da capacidade produtiva dos solos, pois mantêm o C orgânico em níveis adequados (Amado et al., 2001; Beutler et al., 2001) e melhoram a disponibilidade de nutrientes para as culturas subseqüentes (Reicoscky \& Forcella, 1998; Boer et al., 2007). Além disso, outros benefícios podem ser obtidos por essas práticas, tais como: melhoria na estabilidade da estrutura dos solos (Aita et al., 2001), aumento da disponibilidade de P, K, Ca e Mg (Calegari et al., 1992), e melhoria na atividade biológica do solo (Silva et al., 2006a, 2006b).

Ao avaliar o comportamento da mandioca em cultivo mínimo, após a adubação verde com ervilhaca e aveia, Gabriel Filho et al. (2000) verificaram que a produtividade de raízes não foi afetada pelo não revolvimento do solo. Entretanto, Calegari (1993) enfatiza a importância da rotação de culturas, com o uso de plantas de cobertura do solo, para a cultura da mandioca. Essas práticas contribuem para reduzir a ação erosiva da chuva, melhorar a fertilidade natural do solo e reduzir o ataque de pragas e doenças, que é mais intenso quando o cultivo de uma única espécie é realizado sucessivamente na mesma área (Gabriel Filho et al., 2003).

Assim, sistemas de cultivo eficientes, que visem à diminuição do processo erosivo e à recuperação das características físicas, químicas e biológicas do solo, têm sido estudados regionalmente, para obtenção de um maior número de espécies de plantas de cobertura, com potencial de proteção do solo, na cultura da mandioca (Aita et al., 2001). Com este propósito, pesquisas com plantas de coberturas, para uso nas condições edafo-climáticas do Estado de Mato Grosso do Sul, ainda são escassas, o que torna necessária a busca por espécies adaptadas à região e com potencial para contribuir para o aumento da sustentabilidade dos sistemas de produção da cultura da mandioca. Este trabalho teve como objetivo avaliar os efeitos do uso de plantas de coberturas e de sistemas de preparo do solo, no desenvolvimento e produtividade da cultura da mandioca.

\section{Material e Métodos}

O trabalho foi realizado no período de 2002 a 2004, no campo experimental da Prefeitura Municipal de Glória de Dourados, MS $\left(22^{\circ} 22^{\prime} \mathrm{S}\right.$ e $54^{\circ} 30^{\prime} \mathrm{W}$, a $400 \mathrm{~m}$ de altitude), em Argissolo Vermelho de textura arenosa. O clima de ocorrência, conforme Köppen, é do tipo Aw, com estação quente e chuvosa no verão e moderadamente seca no inverno.

Antes do início do trabalho, a área experimental estava sendo utilizada com pastagem de Brachiaria brizantha (Hochst. ex A. Rich.) Stapf. Na safra 2001/2002, foi estabelecida a cultura do milho em sistema convencional, para a homogeneização da área. Este trabalho iniciouse em outubro de 2002, com a divisão da área experimental em quatro talhões de $1.800 \mathrm{~m}^{2}$, onde se efetuou a semeadura de mucuna-cinza (Stizolobium cinereum Piper \& Tracy), sorgo granífero [Sorghum bicolor (L.) Moench] e milheto [Pennisetum americanum (L.) K. Schum.], em preparo convencional, com aração e duas gradagens. No quarto talhão, a área foi deixada em pousio, com posterior preparo para o plantio da mandioca, com aração e duas gradagens (sistema convencional), que serviu como padrão comparativo.

As plantas de cobertura do solo foram dessecadas na floração plena, com glyphosate $\left(3 \mathrm{~L} \mathrm{ha}^{-1}\right)$ e 2,4-D $\left(1 \mathrm{~L} \mathrm{ha}^{-1}\right) \mathrm{e}$, posteriormente, manejou-se a cobertura com um rolo-faca. Após a dessecação, em maio de 2003, efetuou-se o plantio da mandioca, variedade Fécula Branca, em fileira simples, com espaçamento de $0,9 \mathrm{~m}$ entre fileiras e $0,7 \mathrm{~m}$ entre plantas, com aplicação de $456 \mathrm{~kg} \mathrm{ha}^{-1} \mathrm{de}$ fertilizante mineral NPK (4-20-20). A colheita foi realizada em novembro de 2004, aos 18 meses após o plantio.

O delineamento experimental foi o de blocos ao acaso, com seis repetições. Os tratamentos utilizados foram: sistema convencional de preparo de solo (SC), utilizado na área de pousio, e plantio em cultivo mínimo sobre resíduos culturais de mucuna (CMMu), sorgo (CMSo) 
e milheto (CMMi). Cada unidade experimental foi constituída por seis linhas, com dez plantas cada, com área total de $37,8 \mathrm{~m}^{2}$. Considerou-se como área útil os $10,08 \mathrm{~m}^{2}$ de cada parcela, correspondentes à área de 16 plantas, coletadas nas duas linhas centrais.

As variáveis avaliadas foram: altura de plantas, obtida a partir do nível do solo até a extremidade mais alta das plantas, no momento da colheita; produção de matéria seca da parte aérea; número de raízes tuberosas, determinado na ocasião da colheita; produtividade de raízes tuberosas; percentagem de matéria seca (MS) nas raízes tuberosas, determinado pelo método da balança hidrostática, com base na fórmula proposta por Grossmann \& Freitas (1950): MS = 15,75 + 0,0564R, em que $\mathrm{R}$ é o peso de $3 \mathrm{~kg}$ de raízes em água; percentagem de amido nas raízes tuberosas, calculada pela subtração da constante 4,65 do teor de matéria seca (Grossmann \& Freitas, 1950); índice de colheita (IC\%), relação entre a massa de raízes tuberosas e a massa total da planta, de acordo com a fórmula: $\mathrm{IC}=100$ [massa de raízes/(massa de raízes + massa da parte aérea)].

Os resultados dos parâmetros avaliados foram submetidos à análise de variância, e as médias foram comparadas pelo teste de Duncan, a 5\% de probabilidade. Efetuou-se o teste de coeficiente de correlação de Pearson, a 5\%, para todas as variáveis avaliadas.

\section{Resultados e Discussão}

Os parâmetros avaliados variaram significativamente com os tratamentos (Figura 1). Com relação à altura de plantas, observou-se que houve diferença significativa entre os tratamentos, com a maior média observada no CMMi, e o mesmo fato ocorreu para produção de matéria seca da parte aérea, com maiores valores observados no CMMi (Figura 1). Embora não tenha sido quantificada a produção de resíduos nos diferentes tratamentos, verificouse que o milheto produziu maior quantidade de matéria seca e apresentou maior persistência sobre o solo, o que está de acordo com Boer et al. (2007), e pode ter melhorado as condições edáficas neste tratamento, pois fatores como temperatura e cobertura superficial do solo podem disponibilizar mais água às plantas, em solos sob sistema de plantio direto (Salton et al., 1993), com influência direta na economia de água, no desenvolvimento e na produtividade das culturas (Stone \& Silveira, 1999; Bortoluzzi \& Eltz, 2000; Alvarenga et al., 2001).
Observou-se correlação positiva e significativa entre a altura de plantas e a produção da parte aérea $\left(\mathrm{r}=0,68^{*}\right)$, o que está de acordo com Rimoldi et al. (2003). A produção de parte aérea é fator importante na cultura da mandioca, pois está relacionada à produção de material para propagação e com o aproveitamento da cultura como forragem (Vidigal Filho et al., 2000).

No que se refere ao número de raízes tuberosas, observou-se que CMMu e CMMi proporcionaram as maiores médias por hectare, em relação aos demais tratamentos (Figura 1). Segundo Hunt et al. (1977), o número de raízes é definido nos estádios iniciais do ciclo da cultura (dois a três meses) e, assim, a manutenção de condições favoráveis de água e temperatura são fundamentais para a fixação de raízes de armazenamento.

Com relação à produtividade de raízes tuberosas, verificou-se que o CMMi proporcionou maior média (48,6 $\left.\mathrm{Mg} \mathrm{ha}^{-1}\right)$ do que o SC, mas não diferiu dos demais tratamentos (Figura 1). Gabriel Filho et al. (2000) não observaram diferenças significativas, quanto à produtividade de raízes, entre os sistemas de preparo do solo convencional e mínimo, nem entre aveia e ervilhaca utilizadas como plantas de cobertura, o que contrasta com os resultados deste trabalho.

Observou-se correlação positiva e significativa entre número de raízes e massa de matéria seca da parte aérea $(\mathrm{r}=0,72 *)$, entre produtividade de raízes tuberosas e número de raízes $\left(r=0,70^{*}\right)$, e entre altura de planta $\left(\mathrm{r}=0,54^{*}\right)$ e massa de matéria seca da parte aérea $(0,94 *)$. Segundo Cavalcante et al. (2005), o rendimento de raízes e a produtividade de parte aérea de mandioca correlacionam-se positiva e significativamente. O balanço entre parte aérea e raiz, portanto, é importante para a obtenção de altos rendimentos (Viana et al., 2002).

Em relação às características percentagem de matéria seca e de amido nas raízes, os menores valores foram verificados sob preparo convencional do solo, e não foram detectadas diferenças significativas entre os tratamentos com as coberturas vegetais (Figura 1). A cultura da mandioca apresenta, em média, $30 \%$ de matéria seca nas raízes, embora haja registros de até $45 \%$, e o amido varia de 5 a 43\% (Fukuda et al., 2006). Essas características variam muito entre as cultivares e estão ligadas à idade da cultura e ao clima, principalmente chuva e umidade do solo (Toro \& Cañas, 1982). A matéria seca é a característica que determina o maior ou menor rendimento industrial das raízes, uma vez que 

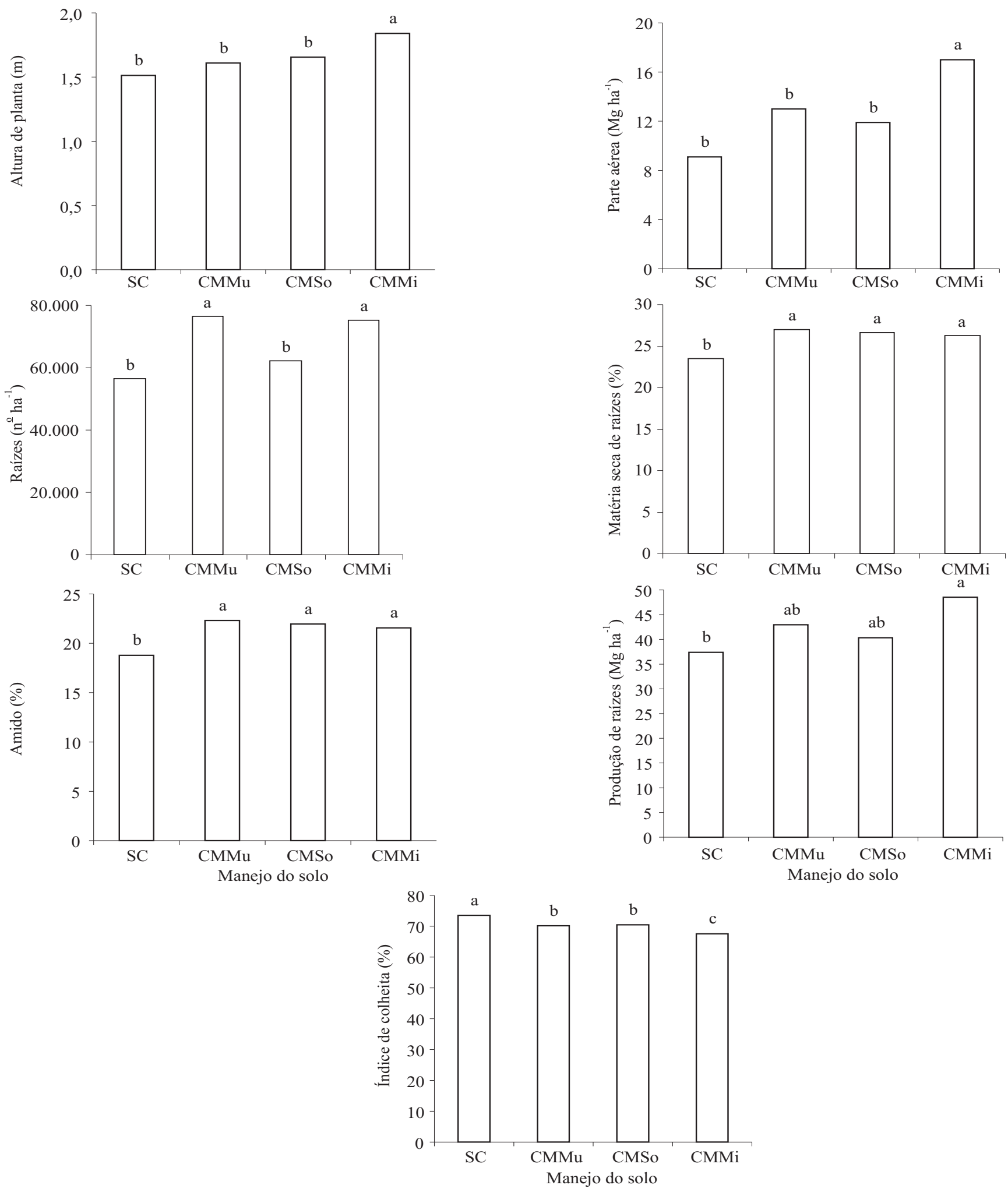

Figura 1. Valores médios de altura de plantas, produção da parte aérea, número de raízes, percentagem de matéria seca de raízes, percentagem de amido de raízes, produção de raízes e índice de colheita de mandioca, cultivada sob diferentes sistemas de manejo de solo: sistema convencional (SC) e plantio direto sobre resíduos de mucuna (CMMu), sorgo (CMSo) e milheto (CMMi). Médias com letras distintas diferem entre si pelo teste de Duncan, a 5\% de probabilidade. 
está diretamente relacionada aos diversos produtos derivados da mandioca (Sarmento, 1997) e é, portanto, desejável que a cultivar mais produtiva seja também aquela que apresente os maiores teores de matéria seca, para maximizar o rendimento do produto final por unidade de área cultivada (Vidigal Filho et al., 2000).

Quanto ao índice de colheita (IC) (relação entre a produção de raízes e a produção total da planta) houve efeito significativo dos tratamentos avaliados (Figura 1). $\mathrm{O}$ tratamento com preparo convencional apresentou o maior valor de IC. O menor valor de IC foi observado no CMMi. Deve-se salientar que os índices de colheita verificados em todos os tratamentos são considerados satisfatórios e não representam limitação para o rendimento da cultura. Segundo Cock \& El-Sharkaway (1991), o valor ótimo para índice de colheita está entre 0,50 e 0,65. Para Conceição (1983), o IC é considerado adequado quando está acima de $60 \%$. O índice de colheita, isoladamente, não fornece informações relevantes sobre o desenvolvimento e produção da planta de mandioca, pois altos valores desse índice podem ser obtidos com o aumento da produção de raízes ou pela diminuição da produção de parte aérea (Cardoso Júnior et al., 2005).

\section{Conclusões}

1. A produção de matéria seca, o número de tubérculos, a produtividade, o índice de colheita e a percentagem de matéria seca e de amido nas raízes de mandioca são influenciados pelo sistema de manejo e preparo do solo.

2. O cultivo mínimo na cultura da mandioca, associado ao uso de plantas de cobertura do solo, promove incrementos na produtividade, quando comparado ao preparo convencional do solo.

\section{Agradecimentos}

Ao Conselho Nacional de Desenvolvimento Científico e Tecnológico e à Fundação de Apoio ao Desenvolvimento do Ensino, Ciência e Tecnologia do Estado do Mato Grosso do Sul, pelo suporte financeiro.

\section{Referências}

AITA, C.;BASSO, C.J.; CERETTA, C.A.; GONÇALVES, C.N.;DAROS, C.O. Plantas de cobertura do solo como fonte de nitrogênio ao milho. Revista Brasileira de Ciência do Solo, v.25, p.157-165, 2001.
ALVARENGA, R.C.; LARA-CABEZAS, W.A.; CRUZ, J.C.; SANTANA, D.P. Plantas de cobertura de solo para sistema plantio direto. Informe Agropecuário, v.22, p.25-36, 2001.

AMADO, T.J.C.; BAYER, C.; ELES, F.L.; BRUM, A.C. Potencial de culturas de coberturas em acumular carbono e nitrogênio no solo no plantio direto e a melhoria da qualidade ambiental. Revista Brasileira de Ciência do Solo, v.25, p.189-197, 2001.

BEUTLER, A.N.; SILVA, M.L.N.; CURI, N.; FERREIRA, M.M.; CRUZ, J.C.; PEREIRA FILHO, I.A. Resistência à penetração e permeabilidade de Latossolo Vermelho distrófico típico sob sistemas de manejo na Região dos Cerrados. Revista Brasileira de Ciência do Solo, v.25, p.167-177, 2001.

BOER, C.A.; ASSIS, R.L. de; SILVA, G.P.; BRAZ, A.J.B.P.; BARROSO, A.L. de L.; CARGNELUTTI FILHO, A.; PIRES, F.R. Ciclagem de nutrientes por plantas de cobertura na entressafra em um solo de cerrado. Pesquisa Agropecuária Brasileira, v.42, p.1269-1276, 2007.

BORTOLUZZI, E.C.; ELTZ, F.L. Efeito do manejo mecânico da palhada de aveia-preta sobre a cobertura, temperatura, teor de água no solo e emergência da soja em sistema de plantio direto. Revista Brasileira de Ciência do Solo, v.24, p.449-457, 2000.

CALEGARI, A. Manejo de adubação verde. In: ENCONTRO NACIONAL DE ROTAÇÃO DE CULTURAS, 2., 1992, Campo Mourão. Ata. Campo Mourão: Associação dos Engenheiros Agrônomos de Campo Mourão, 1993. p.104-116.

CALEGARI, A.; MONDARDO, A.; BULISANI, E.A.; WILDER, L. do P.; COSTA, M.B.B. da; ALCÂNTARA, P.B.; MIYASAKA, S.; AMADO, T.J.C. Adubação verde no Sul do Brasil. Rio de Janeiro: AS-PTA, 1992. 346p.

CARDOSO, C.E.L. Competitividade e inovação tecnológica na cadeia agroindustrial de fécula de mandioca no Brasil. 2003. 188p. Tese (Doutorado) - Escola Superior de Agricultura Luiz de Queiroz, Piracicaba.

CARDOSO JÚNIOR, N.S.; VIANA, A.E.S.; MATSUMOTO, S.N.; SEDIYAMA, T.; CARVALHO, F.M. Efeito do nitrogênio em características agronômicas da mandioca. Bragantia, v.64, p.651-659, 2005.

CAVALCANTE, F.S.; SILVA, I.F.; ARAÚJO, M.C.S.P. Avaliação da viabilidade do consórcio de mandioca e feijão-comum em Latossolo Amarelo no brejo paraibano. Agropecuária Técnica, v.26, p.93-97, 2005.

COCK, J.H.; EL-SHARKAWAY, M.A. Características fisiológicas para la selección de yuca. In: HERSEY, C.H. (Ed.). Mejoramiento genético de la yuca en América Latina. Cali: CIAT: PNDU, 1991. p.257-265.

CONCEIÇÃO, A.J. A mandioca. 2.ed. São Paulo: Nobel, 1983. $382 p$.

FUKUDA, W.M.G.; FUKUDA, C.; VASCONCELOS, O.; FOLGAÇA, J.L.; NEVES, H.P.; CARNEIRO, G.T. Variedades de mandioca recomendadas para o Estado da Bahia. Bahia Agrícola, v.7, p.27-30, 2006.

GABRIEL FILHO, A.; PESSOA, A.C.S.; STROHHAECKER, L.; HELMICH, J.J. Preparo convencional e cultivo mínimo do 
solo na cultura da mandioca em condições de adubação verde com ervilhaca e aveia. Ciência Rural, v.30, p.953-957, 2000.

GABRIEL FILHO, A.; STROHHAECKER, L.; FEY, E. Profundidade e espaçamento da mandioca no plantio direto na palha. Ciência Rural, v.33, p.461-467, 2003.

GROSSMANN, J.; FREITAS, A.C. Determinação do teor de matéria seca pelo peso específico em raízes de mandioca. Revista Agronômica, v.14, p.75-80, 1950.

HUNT, L.A.; WHOLEY, D.W.; COCK, J.H. Growth physiology of cassava (Manihot esculenta Crantz). Field Crop Abstracts, v.30, p.77-91, 1977.

REICOSKY, D.C.; FORCELLA, F. Cover crop and soil quality interactions in agroecosystems. Journal of Soil and Water Conservation, v.53, p.224-229, 1998.

RIMOLDI, F.; VIDIGAL FILHO, P.S.; SCAPIM, C.A.; VIDIGAL, M.C.G. Avaliação de cultivares de mandioca nos municípios de Maringá e Rolândia no Estado do Paraná. Acta Scientiarum: Agronomy, v.25, p.459-465, 2003.

RIMOLDI, F.; VIDIGAL FILHO, P.S.; VIDIGAL, M.C.G.; CLEMENTE, E.; PEQUENO, M.G.; MIRANDA, L.; KVITSCHALL, M.V. Produtividade, composição química e tempo de cozimento de cultivares de mandioca-de-mesa coletadas no Estado do Paraná. Acta Scientiarum: Agronomy, v.28, p.63-69, 2006.

SALTON, J.C.; PITOL, C.; ERBES, E.J. Cultivos de primavera: alternativas para produção de palha em Mato Grosso do Sul. Maracaju: Fundação MS, 1993. 6p. (Fundação MS. Informativo técnico, 1).

SARMENTO, S.B.S. Caracterização da fécula de mandioca (Manihot esculenta, Crantz) no período de colheita de cultivares de uso industrial. 1997. 162p. Tese (Doutorado) - Universidade de São Paulo, São Paulo.
SILVA, R.F.; AQUINO, A.M.; MERCANTE, F.M.; GUIMARÃES, M.F. Macrofauna invertebrada do solo sob diferentes sistemas de produção em Latossolo da Região do Cerrado. Pesquisa Agropecuária Brasileira, v.41, p.697-704, 2006a.

SILVA, R.F.; AQUINO, A.M.; MERCANTE, F.M.; GUIMARÃES, M.F. Populações de oligoquetos (Annelida: Oligochaeta) em um Latossolo Vermelho submetido a sistemas de uso de solo. Ciência Rural, v.36, p.673-677, 2006b.

SOUZA, A.B.; FASIABEN, M.C.R. Competição de cultivares de mandioca conduzida em uma pequena propriedade no Município de Rio Azul, Paraná. Revista Brasileira de Mandioca, v.5, p.99-104, 1986.

SOUZA, L.D.; SOUZA, L.S.; GOMES, J.C. Exigências edáficas da cultura da mandioca. In: SOUZA, L.S.; FARIAS, A.R.N.; MATTOS, P.L.P.; FUKUDA, W.M.G. (Ed.). Aspectos socioeconômicos e agronômicos da mandioca. Cruz das Almas: Embrapa Mandioca e Fruticultura Tropical, 2006. p.70-214.

STONE, L.F.; SILVEIRA, P.M. Efeito do sistema de preparo na compactação do solo, disponibilidade hídrica e comportamento do feijoeiro. Pesquisa Agropecuária Brasileira, v.34, p.83-91, 1999.

TORO, J.C.; CAÑAS, A. Determinación del contenido de materia seca y almidón en yuca por el sistema de gravedad específica. In: DOMINGUEZ, C.E. (Comp.). Yuca: investigación, producción y utilización. Cali: CIAT, 1982. p.567-575.

VIANA, A.E.S.; SEDIYAMA, T.; LOPES, S.C.; CECON, P.R.; SILVA, A.A. Avaliação de métodos de preparo de manivas de mandioca (Manihot esculenta Crantz). Ciência e Agrotecnologia, p.1383-1390, 2002. (Edição especial).

VIDIGAL FILHO, P.S.; PEQUENO, M.G.; SCAPIM, C.A.; VIDIGAL, M.C.G.; MAIA, R.R.; SAGRILO, E.; SIMON, G.A.; LIMA, R.S. Avaliação de cultivares de mandioca na Região Noroeste do Paraná. Bragantia, v.59, p.69-75, 2000.

Recebido em 8 de outubro de 2007 e aprovado em 20 de março de 2008 